\title{
Bridging the quality gap in diabetes care
}

\author{
Sheena Mc Hugh \\ Department of Epidemiology \& Public Health, School of Medicine, UCC
}

"At the end of the day it's not about me and it's not about the hospital, it's about the patient." The sentiment of this GP was echoed by many others during interviews which I conducted to better understand attitudes towards improving diabetes care. Ultimately, the purpose of the health system is to improve the health and quality of life of the patient, not the system itself. This goal appears obvious and yet a gap remains between the ideal, as set out in national and international guidelines, and the reality of care delivered to people with diabetes.

\section{The Quality Gap}

The gap in quality is by no means a distinctly Irish problem and we can certainly learn from the efforts of other countries. However the techniques we choose to improve the quality of care will need to be adapted to fit our national context and local circumstances. With this in mind, I began my research by surveying a national sample of GPs to examine how care is currently provided in general practice. Registering, reviewing and recalling patients are the 3 pillars of a comprehensive diabetes service. Only half of GPs surveyed were routinely recalling patients with diabetes to review their health while less than half were using a patient register. There was also deficient access to necessary services to support diabetes care such as dietetic services, chiropody and podiatry services and retinal screening. Thus little progress had been made towards structuring diabetes care in general practice since the last survey conducted in 2003, before the establishment of the HSE.

\section{Building a bridge}

Quality improvement interventions usually employ several different methods to enhance care. Some of the methods which have proved popular and effective include auditing care and providing feedback to health care professionals, developing a computerised recall system to help track patients and enhancing the role of the nurse. However no one strategy has proved to be the missing ingredient. In fact quality improvement interventions usually include so many ingredients it is difficult to make conclusions about what works for whom. What all of the interventions have in common is their aim to improve quality and their attempt to structure and organise care delivery albeit by different means. 
Ireland has a number of "islands of excellence" when it comes to improving the delivery of diabetes care. These "pockets of interest", as one GP put it, arose out of both necessity and interest in different areas around Ireland. Having worked with three such groups in the East, Midlands and South of Ireland, we demonstrated the effectiveness of adopting a structured approach to diabetes care in general practice. This is the 1st national study of structured care in the Irish community and combines data from over 3000 patients with Type 2 diabetes. We looked at how care was delivered in the practice (i.e. process of care) and also how well these patients 'do' having received structured care (i.e. outcome of care). The majority of patients were receiving a high standard of care with outcomes such as metabolic control, blood pressure and cholesterol being checked and recorded by the GP on a regular basis. Patients were also doing well. For example the average cholesterol level among patients was $4.1 \mathrm{mmol} / 1$ meeting the standard of less than $4.5 \mathrm{mmol} / 1$ set out in the guidelines. Not only are patients doing well by our national standards, they are also doing well compared to results from the UK.

However there is always room for improvement! Lifestyle factors such as exercise and diet need ongoing attention both from doctors and patients. Half of all the patients enrolled in structured care fell into the obese BMI category $(<30 \mathrm{~kg} / \mathrm{m} 2)$. Overweight and obesity continues to be a major challenge in the provision of diabetes care as this patient group are at increased risk of cardiovascular complications and cardiovascular disease mortality. Consequently there is a growing need to collect information on the long-term complications associated with diabetes including heart problems but also blindness, foot amputations, and renal failure. Only then can we be certain that there has been a lasting improvement.

\section{Getting over it}

And yet if we know our destination why have we not reached it? This is one of the fundamental questions driving my research. From my interviews with GPs it seems the barriers to improvement occur at many levels. At the health system level, the lack of integration between the general practice and hospital settings creates a barrier which impacts on the delivery of care, the providers and the patients causing "a big palaver". For example "if you check the cholesterol, get it to the patient to bring to the hospital and it gets lost in the process lots of times and then it seems incredibly wasteful of effort and time and resources to repeat it a few times". Some GPs felt there was a lack of resources, remuneration and recognition for chronic disease management. Hence at a professional level, diabetes care becomes a "labour of love" for many GPs around Ireland. It is this "vocational" incentive which is currently driving our "pockets of interest". However the lack of recognition could lead to "bad feeling" among health care providers and leave them indifferent and apathetic to future change. GPs were sceptical about future improvements 


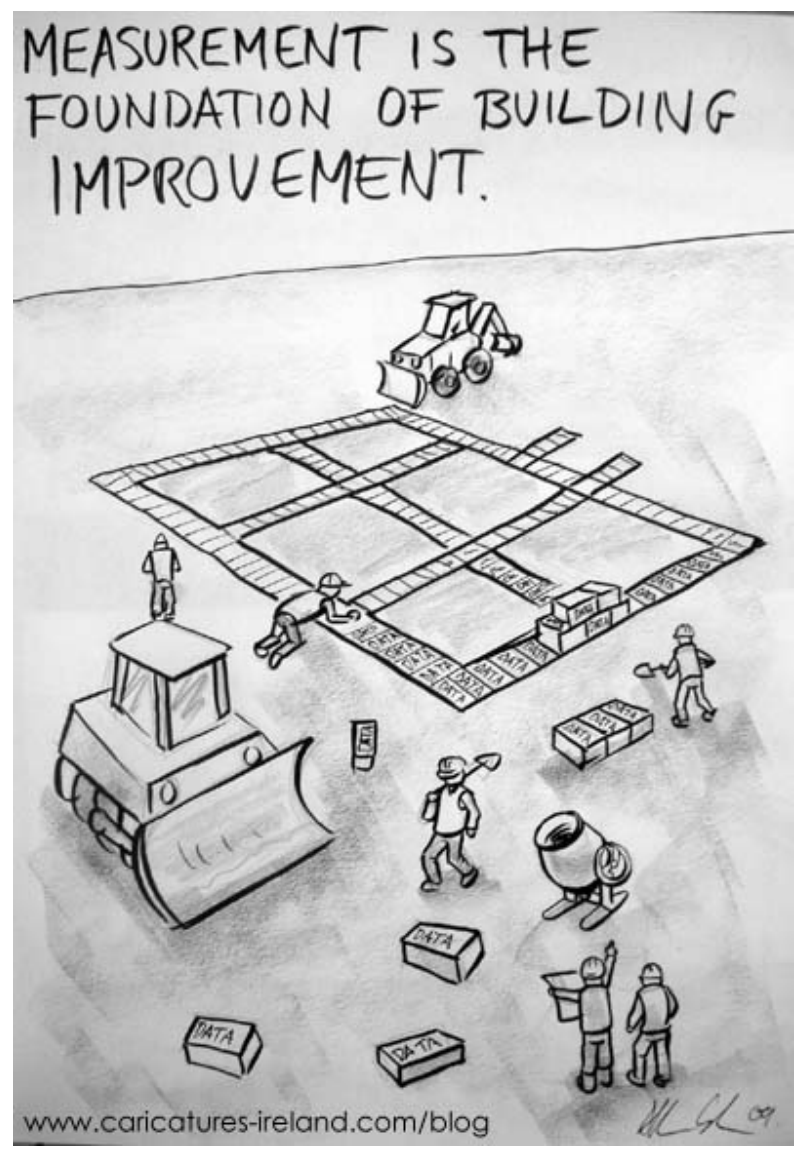

based on empty promises and dashed hopes in the past.

It's an idealistic approach, and I'm afraid, in my lifetime, anything idealistic has never come to fruition... Not even once has the idea ever come into play. In practical terms, they never work, and haven't worked in the past... but that doesn't mean you should stop hoping for it.

\section{Conclusions}

Without sounding too much like Barack Obama, there are opportunities to change and improve diabetes care. Those with a special interest are pioneering a structured approach to care and producing positive results which suggests the barriers are not insurmountable. However, this level of interest is not sustainable in the face of increasing demands and dwindling resources. The challenge now becomes creating a national infrastructure which supports local efforts to cross the quality gap to avoid a situation whereby the quality of care received by patients is determined by geography.

Sheena Mc Hugh is a student in the Health Services Research Institute- the Irish Health Research Board's Cross-Institution PhD Scholar Programme in Health Services Research under the supervision of Prof. Ivan J. Perry (UCC), Prof. Colin Bradley (UCC) and Prof. Ruairi Brugha 
(Royal College of Surgeons). The author would like to acknowledge the Health Research Board for funding this research. Credit is also extended to Allan Cavanagh for permission to use the cartoon from caricatures-ireland.com/blog. 